\title{
Use of four genes in exosomes as biomarkers for the identification of lung adenocarcinoma and lung squamous cell carcinoma
}

\author{
BINGJI CAO ${ }^{1}$, PENGYU WANG ${ }^{2}$, LINA GU $^{3}$ and JUNFENG LIU ${ }^{1}$ \\ Departments of ${ }^{1}$ Thoracic Surgery and ${ }^{2}$ Clinical Laboratory, ${ }^{3}$ Research Center, \\ The Fourth Hospital of Hebei Medical University, Shijiazhuang, Hebei 050011, P.R. China
}

Received August 21, 2020; Accepted January 82021

DOI: $10.3892 / \mathrm{ol} .2021 .12510$

\begin{abstract}
The determination of biomarkers in the blood specific for lung adenocarcinoma (LUAD) and lung squamous cell carcinoma (LUSC) is crucial for the selection of effective treatment strategies and the prediction of prognosis. The purpose of the present study was to analyze the differentially expressed genes (DEGs) in LUSC and LUAD from The Cancer Genome Atlas (TCGA) database. In order to identify the potential biomarkers for non-small cell lung cancer (NSCLC) for clinical diagnosis, bioinformatics was used to analyze the DEGs of two subtypes of NSCLC, LUAD and LUSC. Exosomes were isolated from the serum of patients with LUAD or LUSC and identified using transmission electron microscopy, nanoparticle tracking analysis and western blot analysis. A total of four differential exosomal mRNAs were selected for validation with serum samples from 70 patients with NSCLC via reverse transcription-quantitative polymerase chain reaction. Receiver operating characteristic curves were established to evaluate the clinical diagnostic value of four DEGs for patients with LUAD and LUSC. The analysis based on TCGA data revealed the DEGs in LUSC and LUAD: A total of 1,619 genes were differentially expressed in patients with LUSC and LUAD. DEGs analyzed by Gene Ontology and Kyoto Encyclopedia of Genes and Genomes enrichment analyses revealed that inflammation-related signaling pathways, such as complement pathways, and multiple autoimmune diseases, such as systemic lupus erythematosus and asthma were mainly enriched in LUAD. The cell cycle, Hippo signaling pathway, Rap1 signaling pathway and Wnt signaling pathway were the main signaling pathways enriched in LUSC. The combination of tumor protein P63 (TP63), keratin 5 (KRT5), CEA cell adhesion molecule 6 (CEACAM6)
\end{abstract}

Correspondence to: Dr Junfeng Liu, Department of Thoracic Surgery, The Fourth Hospital of Hebei Medical University, 12 Jiankang Road, Shijiazhuang, Hebei 050011, P.R. China E-mail: liujf@hbmu.edu

Key words: lung adenocarcinoma, lung squamous cell carcinoma, exosomes, tumor protein P63, keratin 5, CEA cell adhesion molecule 6 and surfactant protein $B$ and surfactant protein B (SFTPB) improved the specificity and sensitivity in the diagnosis of different lung cancer subtypes. Exosomal TP63, KRT5, CEACAM6 and SFTPB mRNAs can thus be used as biomarkers to differentiate between LUSC and LUAD, and may provide a novel strategy for their differential diagnosis and treatment.

\section{Introduction}

Lung cancer is the most common type of tumor and is has the highest mortality rate worldwide (1). The incidence rate of lung cancer was $11.6 \%$ and mortality rate was $18.4 \%$ worldwide in 2018 (1). The majority of patients with lung cancer $(>80 \%)$ are diagnosed with non-small cell lung cancer (NSCLC) (2). There are two main subtypes of NSCLC: Lung adenocarcinoma (LUAD) and lung squamous cell carcinoma (LUSC) $(3,4)$. Different subtypes of NSCLC are related to different molecular biological characteristics $(5,6)$. LUAD, for example, arising from the distal airway, is often associated with the mutation of the KRAS gene. By contrast, LUSC, occurring in the proximal airway, is often associated with the deletion of chromosome $3 p(7,8)$. To date, limited information is available on the molecular variations between the two subtypes, which are considered to be the reason for the different responses to treatment. Patients with LUSC survive longer compared with those with LUAD following treatment with ipilimumab (9), while patients with LUAD most likely benefit from the use of gefitinib, a drug targeting epithelial growth factor receptor (EGFR) kinase mutation (10). These differences in tumor biology and drug response suggest the importance of accurately distinguishing LUAD from LUSC. Recently, it has been found that mRNAs can distinguish LUSC from LUAD (11); thus, the determination of differential gene expression may aid in distinguishing between the two subtypes.

Exosomes are extracellular vesicles secreted by cells; they exist in urine, plasma, lavage fluid, serous cavity effusion, cerebrospinal fluid and other body fluids $(12,13)$. They have a lipid bilayer with a diameter of 30-150 nm, and their contents include DNA, RNA, protein and lipids $(14,15)$. Exosomes are released through cells into the circulation and body fluids. Studies have demonstrated that exosomes have different protein and RNA contents in healthy subjects and patients with cancer, which provides the basis for their use as diagnostic markers (16). However, it remains unknown whether the 
aforementioned molecular diagnostic markers are carried by exosomes in patients with NSCLC, and whether they can be used to distinguish between LUSC and LUAD.

The purpose of the present study was to analyze the differentially expressed genes (DEGs) in LUSC and LUAD from The Cancer Genome Atlas (TCGA) database. mRNAs were identified as potential molecular diagnostic markers to distinguish LUSC and LUAD using bioinformatics analyses, and these molecular markers were verified using exosomes from the serum of patients with NSCLC.

\section{Materials and methods}

Microarray datasets. Gene expression profile analysis data were obtained from TCGA database (https://portal.gdc.cancer. gov/). The data of LUSC and LUAD tissues were used in the present study. The microarray data included 504 cases of LUSC and 522 cases of LUAD. EdgeR-3.30.0 software was used to analyze the DEGs (17). The corrected P-value was obtained for the false discovery rate (FDR) using the Benjamini and Hochberg $(\mathrm{BH})$ method. mRNAs with FDR $<0.01$, fold change $>2$ and median of trans per million (TPM) $>5$ were defined as having statistically significant differential expression. According to the National Center for Biotechnology Information database (https://www.ncbi.nlm.nih.gov), genes corresponding to these mRNAs were identified.

Bioinformatics analysis. The present study used the Database for Annotation, Visualization and Integrated Discovery (DAVID) bioinformatics web server (https://david.ncif crf. gov/tools.jsp/) as a tool to explore the potential function of differentially expressed DEGs by performing Gene Ontology (GO; www.geneontology.org/) and Kyoto Encyclopedia of Genes and Genomes (KEGG) pathway-enrichment analysis. GO functional enrichment analysis of DEGs was performed using the GO online database (http://www.geneontology.org). KEGG database (http://www.genome.jp/kegg) was used to analyze the functions involved in the pathways. The P-value indicates the importance of pathways and related genes. According to the order of the P-value, the terms/pathways with $\mathrm{P}<0.01$ were selected for the network. As the number of terms/pathway-related genes was countless, the data were filtered to produce the network, with 30 terms/pathways maintained for each network. According to the specific standard: Due to the large number of genes screened out, 30 genes with the most obvious changes were chosen and GO analysis and KEGG analysis performed. if there were too many genes $(>30)$ with $\mathrm{P}<0.01$, the threshold $\mathrm{P}$-value was further reduced $(\mathrm{P}<0.001$ or $\mathrm{P}<0.0001$ and so on). The final filtered terms/pathways included $\sim 30$ genes, or $<30$ genes.

Clinical patients. A total of 16 patients with LUSC and 54 patients with LUAD were recruited from the Department of Thoracic Surgery, Fourth Hospital of Hebei Medical University (Shijiazhuang, China) between October 2017 and October 2018. There were 38 males and 32 females (mean age \pm SD, $65 \pm 9.8$ years; age range, $54-73$ years). The inclusion criteria were as follows: i) All patients were diagnosed with LUSC or LUAD by histopathological or cytological examination. The exclusion criteria were as follows: i) Patients with multiple types of cancer; ii) patients with the tumor site originating from the lung; iii) patients with pulmonary metastasis; iv) patients with hemolysis, hyperlipidemia and other abnormal blood diseases; v) and patients who had received radiotherapy or chemotherapy. The samples were collected from the Department of Thoracic Surgery of the Fourth Hospital of Hebei Medical University. The study was approved by the Ethics Committee of the Fourth Hospital of Hebei Medical University. Informed consent was signed by all patients or their families.

Blood collection and separation of plasma exosomes. A total of $5 \mathrm{ml}$ venous blood samples were collected from all subjects, centrifuged within $4 \mathrm{~h}$ at $3,000 \mathrm{x}$ g for $15 \mathrm{~min}$ at $4^{\circ} \mathrm{C}$, and the serum on the upper layer was collected. Hemolysis was avoided during the whole process, if hemolysis was found, samples were collected again. The samples were labeled and stored at $-80^{\circ} \mathrm{C}$. The separation of exosomes was performed by ultracentrifugation, as previously described (18). The serum samples were thawed on ice and centrifuged at $4^{\circ} \mathrm{C}$ for $30 \mathrm{~min}$ at $10,000 \mathrm{x} \mathrm{g}$. The samples were then centrifuged at $100,000 \mathrm{x} \mathrm{g}$ at $4^{\circ} \mathrm{C}$ for $120 \mathrm{~min}$. The precipitate was then resuspended in 2-3 $\mathrm{ml} 1 \mathrm{X}$ PBS and filtered using a $0.22-\mu \mathrm{m}$ aperture filter. The mixture was centrifuged again for $120 \mathrm{~min}$ at $100,000 \mathrm{x} \mathrm{g}$ at $4^{\circ} \mathrm{C}$.

Transmission electron microscopy (TEM). A $10 \mu \mathrm{l}$ exosomes solution was placed on a copper mesh and incubated at room temperature for $1 \mathrm{~min}$. After washing with sterile distilled water, the exosome was contrasted by uranyl acetate solution for $1 \mathrm{~min}$. The sample was then dried for $2 \mathrm{~min}$ under incandescent light. The copper mesh was observed and photographed under a transmission electron microscope (H-7650; Hitachi Ltd.).

Nanoparticle tracking analysis (NTA). The measurement of exosome size and particle concentration was performed according to the manufacturer's protocol using a ZetaView PMX 110 (Particle Metrix GmbH). Izon Control Suite software v.3.3.2.2000 (Izon Science Ltd.) was used to analyze the data.

Western blot analysis. Total exosome protein was extracted from the exosome samples using RIPA lysis buffer (Invitrogen; Thermo Fisher Scientific, Inc.) with PMSF protease inhibitor (Invitrogen; Thermo Fisher Scientific, Inc.). The protein quality of samples were calculated by the BSA standard protein solutions curve with PierceTM BCA Protein Assay Kit (cat. no. 23,225; Thermo Fisher Scientific Inc.). The samples (30 $\mu \mathrm{g}$ per lane) were separated via SDS-PAGE (12\% gel) for the western blot analysis. The proteins were transferred to PVDF membranes and blocked with evaporated skimmed milk for $1 \mathrm{~h}$ at $37^{\circ} \mathrm{C}$ and incubated overnight at $4^{\circ} \mathrm{C}$ with primary antibodies. The membranes were then incubated for $2 \mathrm{~h}$ at $37^{\circ} \mathrm{C}$ with sheep anti-rabbit secondary antibody conjugated with HRP (1:10,000; cat. no. RS0002; ImmunoWay Biotechnology) or sheep anti-mouse secondary antibody conjugated with HRP (1:10,000; cat. no. RS0001; ImmunoWay Biotechnology). The protein bands were exposed using ECL blotting detection reagents (cat. no. P0018; Beyotime 
Institute of Biotechnology). The primary antibodies were as follows: Anti-CD63 [1:1,000; cat. no. sc-5275 (M), Santa Cruz Biotechnology, Inc.], anti-ALG-2 interacting protein $\mathrm{X}$ (1:1,000; ALIX; cat. no. sc-53540; Santa Cruz Biotechnology, Inc.), anti-calnexin (1:1,000; cat. no. 10427-2-AP; ProteinTech Group, Inc.) and anti-tumor susceptibility gene 101 protein (1:1,000; TSG101; cat.no. sc-136111; Santa Cruz Biotechnology, Inc.) were used for western blot analysis. Calnexin was the exosome-negative marker.

$R N A$ isolation and reverse transcription-quantitative PCR $(R T-q P C R)$. Following the suspension of exosomes with $1 \mathrm{ml}$ PBS, total RNA was extracted from the exosomes using TRIzol ${ }^{\circledR}$ reagent (Invitrogen; Thermo Fisher Scientific, Inc.). The RNA concentration was measured using a Nanodrop 2000 UV-Vis spectrophotometer (Nanodrop Technologies; Thermo Fisher Scientific, Inc.). A total of $1 \mu \mathrm{g}$ total RNA was reverse transcribed into cDNA according to the manufacturer's protocol by using the PrimeScript ${ }^{\mathrm{TM}}$ RT reagent kit (cat. no. RR037A; Takara Bio Inc.). qPCR was then performed using Premix Ex Taq ${ }^{\mathrm{TM}}$ reagent (cat. no. RR390A; Takara Bio, Inc.). The thermocycling conditions were as follows: Predenaturation at $95^{\circ} \mathrm{C}$ for $5 \mathrm{~min}$, denaturation at $95^{\circ} \mathrm{C}$ for $15 \mathrm{sec}$, annealing at $58^{\circ} \mathrm{C}$ for $30 \mathrm{sec}$, extension at $72^{\circ} \mathrm{C}$ for $30 \mathrm{sec}$, a total of 40 cycles; extension at $72^{\circ} \mathrm{C}$ for $10 \mathrm{~min}$. GAPDH was used as an internal control. The relative expression levels of DEGs in serum samples were evaluated using the $2^{-\Delta \Delta \mathrm{Cq}}$ method (19). The primer sequences are listed in Table I. All experiments were repeated three times. RT-qPCR analysis is highly sensitive, and its accuracy can be affected by RNA quantity, transcription efficiency, amplification efficiency and experimental procedures between samples (20). To avoid bias, normalization of gene expression is an essential step (20). The most common practice is to compare a target gene expression with an internal reference gene (21). Housekeeping genes, such as $\beta$-actin (ACTB), glyceraldehyde-3-phosphate dehydrogenase (GAPDH), and solute carrier family 25 member 6 (SLC25A6) (22-24) have been used extensively for RT-qPCR analysis. However, under any given experimental condition, the expression of these commonly used reference genes may vary substantially $(25,26)$. In order to ensure the accuracy of the results, $\beta$-actin (ACTB) and solute carrier family 25 member 6 (SLC25A6) were used as internal references in the present study.

Statistical analysis. All data are analyzed using SPSS (version 22.0; IBM Corp.) and GraphPad Prism (version 6.0; GraphPad Software, Inc.) software. EdgeRv.3.30.0 software was used to analyze the DEGs (17). The P-value was corrected for FDR using the Benjamini and Hochberg (BH) method (27). mRNAs with FDR $<0.01$, fold change $>2$ and a median TPM $>5$ were defined as having a statistically significant differential expression. Fisher's exact test was used to calculate the significance (P-value) of the GO and KEGG enrichment analyses. The means of the expression of four DEGs in the LUSC and LUAD group were compared using an independent t-test. The association between gene expression and clinical biological parameters was analyzed using the Chi-square test or Fisher's test. The logistic regression model of generalized linear models ( $\mathrm{R}$ programming language) was used to model
Table I. Sequences of the primers.

\begin{tabular}{lc}
\hline Gene & Sequence \\
\hline TP63 & \\
Forward & 5'-CTCCAACACCGACTACCCAG-3' \\
Reverse & 5'-GCGGATAACAGCTCCCTGAG-3' \\
KRT5 & \\
Forward & 5'-GGGCGAGGAATGCAGACTC-3' \\
Reverse & 5'-ACTGCCATATCCAGAGGAAACA-3' \\
CEACAM6 & \\
Forward & 5'-TCTTGTGAATGAAGAAGCAACCG-3' \\
Reverse & 5'-CACAGCATCCTTGTCCTCCA-3' \\
SFTPB & \\
Forward & 5'-GCTGGACAGGGAAAAGTGC-3' \\
Reverse & 5'-TGGATACACTGGAGAGGGCT-3' \\
ACTB & \\
Forward & 5'-CCTCGCCTTTGCCGATCC-3' \\
Reverse & 5'-CATGCCCACCATCACGC-3' \\
SLC25A6 & \\
Forward & 5'-GGCCTACTTCGGCGTGTAC-3' \\
Reverse & 5'-CGAAGGGGTAGGACACCACG-3' \\
\hline
\end{tabular}

TP63, tumor protein P63; KRT5, keratin 5; CEACAM6, CEA cell adhesion molecule 6; SFTPB, surfactant protein B; ACTB, $\beta$-actin; SLC25A6, solute carrier family 25 member 6 .

the $\Delta \mathrm{Cq}$ data of 70 cases, and the single gene and four gene combinations were analyzed, respectively. A receiver operating characteristic (ROC) curve was drawn for each model and the area under the curve (AUC) of the model was calculated to evaluate the performance. $\mathrm{P}<0.05$ was considered to indicate a statistically significant difference.

\section{Results}

DEGs of LUSC and LUAD. In order to determine the specific mRNA profiles of serum exosomes in patients with LUSC and LUAD, 1,026 patients with NSCLC, including 504 LUSCs and 522 LUADs from TCGA database were analyzed using bioinformatics. The purpose of this study was to analyze the DEGs of LUSC and LUAD, and explore these genes in order to distinguish between LUSC and LUAD. Therefore, samples from healthy individuals or adjacent non-cancerous samples were not used. Following the data analysis, a total of 1,619 genes were identified as differentially expressed in patients with LUSC and LUAD. Considering that the markers in tissues are diluted by the RNA from other tissue sources when they are detected in blood, the markers with a high expression were selected. In the present study, 17 DEGs with a median TPM >1,000 were selected from the LUSC and LUAD group. Furthermore, hierarchical cluster analysis was applied to the 17 DEGs (Fig. 1). Among these, eight of the 17 DEGs were upregulated in the LUSC and downregulated in the LUAD group; nine of the 17 DEGs were upregulated in the LUAD and downregulated in the LUSC group (Table II) (28-43). 


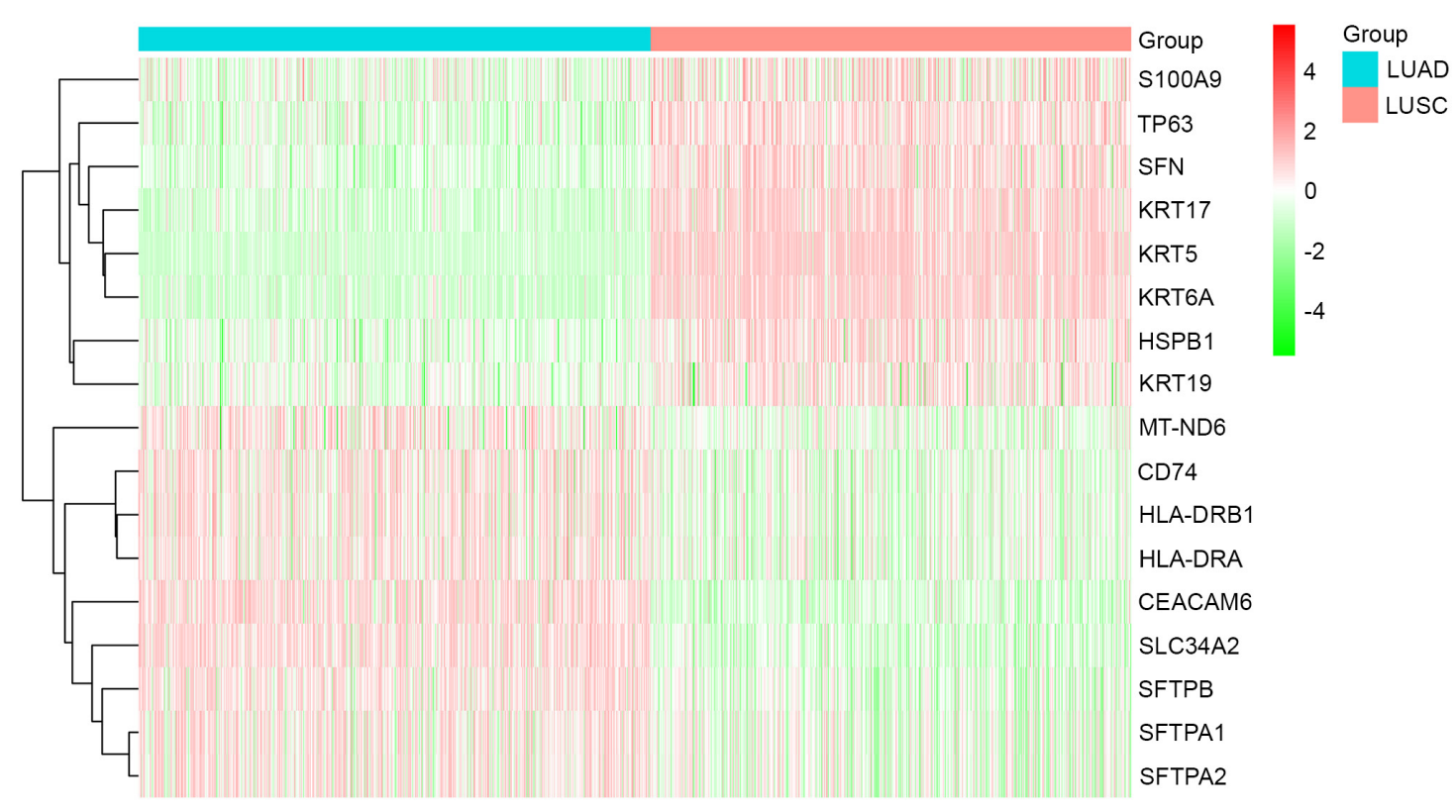

Figure 1. Cluster analysis of 17 types of differentially expressed mRNAs based on The Cancer Genome Atlas database. Red color represents upregulation and green represents downregulation. LUAD, lung adenocarcinoma; LUSC, lung squamous cell carcinoma.

Biological function and signaling pathway enrichment analysis of DEGs. The DEGs of LUAD and LUSC were analyzed using the DAVID database (https://david.ncif crf.gov/tools.jsp/). DEGs in LUAD were mainly enriched in multiple biological processes, such as the 'regulation of complement activation', 'signaling pattern recognition receptor activity', 'pattern recognition receptor signaling pathway' and the 'negative regulation of epithelial-to-mesenchymal transition', 'BMP receptor binding' and 'tight junction' (Fig. 2A). However, DEGs in LUSC were mainly enriched in multiple biological processes, such as 'cell division', 'mitotic nuclear division', the ' $\mathrm{G}_{1} / \mathrm{S}$ transition of mitotic cell cycle', 'mitotic sister chromatid segregation', 'spindle pole' and 'spindle microtubule' (Fig. 2B). Complement activation is an important early event in the inflammatory response. However, DEG enrichment in LUAD was higher compared with that of DEGs in LUSC in the complement activation pathway, indicating that there was a more prominent association with the inflammatory response.

To investigate the important ways of DEGs, KEGG pathway analysis was performed. According to the expression of DEGs, three signaling pathways with the highest enrichment in LUAD were 'complement and coagulation cascades', 'Staphylococcus aureus infection', 'cell adhesion molecules' and 'tight junction' (Fig. 3A). However, three signaling pathways with the highest enrichment in LUSC were the cell cycle, p53 signaling pathway and pathways in cancer (Fig. 3B).

Identification of exosomes. The characteristics of exosomes were identified by TEM, western blot analysis and NTA, and the plasma exosomes of one patient with lung cancer (Fig. 4A) were observed under a transmission electron microscope. It was observed that the structure of plasma exosomes included a lipid bilayer membrane and had a size $<200 \mathrm{~nm}$. In addition, Alix, TSG101 and CD63, protein markers of plasma-derived exosomes, were observed in exosomes and the whole cell extract, whereas calnexin was the exosome-negative marker.
There were two bands of Alix in the whole cell lysate, with the top band indicating non-specific staining. As the protein modification level of TSG101 in different types of samples differs, the whole cell lysate band was smaller compared with that of the plasma exosome, which is a normal phenomenon (36). Due to the different glycosylation levels of CD63, the bands were diffuse with a fragment size of $30-60 \mathrm{kDa}(44)$. Therefore, the protein bands of CD63 in exosomes were shallower, but were also distributed in this region (30-60 kDa). Due to the antibody used, calnexin presented two bands with the lower band corresponding to calnexin (band size, $90 \mathrm{kDa}$ ) and the upper $120 \mathrm{kDa}$ band being non-specific (Fig. 4B). Furthermore, NTA revealed that the diameter of the exosomes was $25-235 \mathrm{~nm}$, enriched at $99.2 \mathrm{~nm}$ and the concentration was $\sim 4.2 \times 10^{7}$ particles $/ \mathrm{ml}$ (Fig. 4C). In addition, Alix, TSG101 and CD63, protein markers of plasma-derived exosomes, were observed in exosomes and the whole cell extract, whereas Calnexin was the exosome-negative marker. There were two bands of Alix in the whole cell lysate, with the top band indicating non-specific staining. As the protein modification level of TSG101 in different types of samples differs, the whole cell lysate band was smaller compared with that of the plasma exosome, which is a normal phenomenon (36). Due to the different glycosylation levels of CD63, the bands were diffuse with a fragment size of $30-60 \mathrm{kDa}$ (44). Therefore, the protein bands of CD63 in exosomes were shallower, but were also distributed in this region $(30-60 \mathrm{kDa})$. Due to the antibody used, calnexin presented two bands with the lower band corresponding to calnexin (band size, $90 \mathrm{kDa}$ ) and the upper $120 \mathrm{kDa}$ band being non-specific (Fig. 4C).

Exosomal tumor protein P63 (TP63), keratin 5 (KRT5), CEA cell adhesion molecule 6 (CEACAM6) and surfactant protein B (SFTPB) $m R N A$ as biomarkers for distinguishing $L U A D$ from LUSC. To confirm the feasibility of these genes in differentiating LUAD from LUSC, TP63, KRT5, CEACAM6 


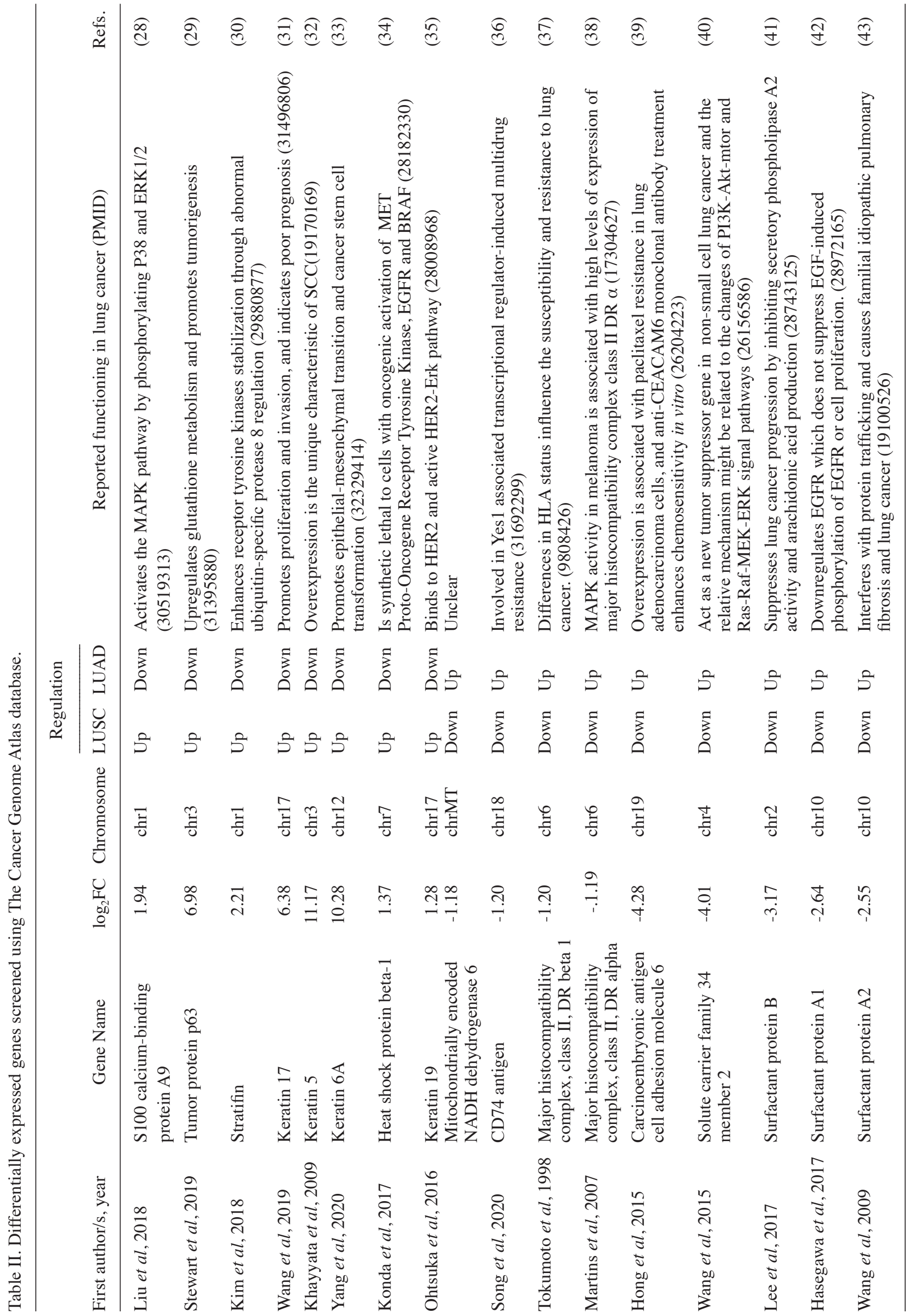


A

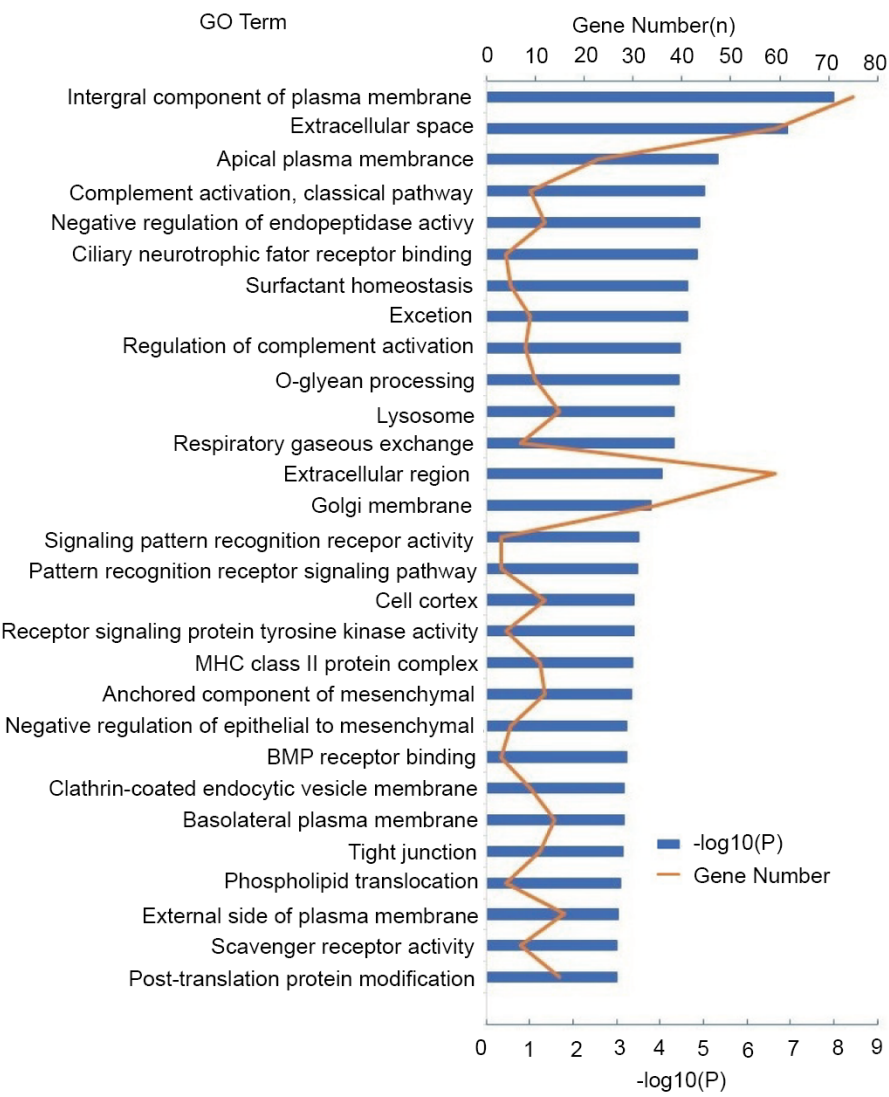

B Go Term Gene Number(n)

020406080100120140160180200

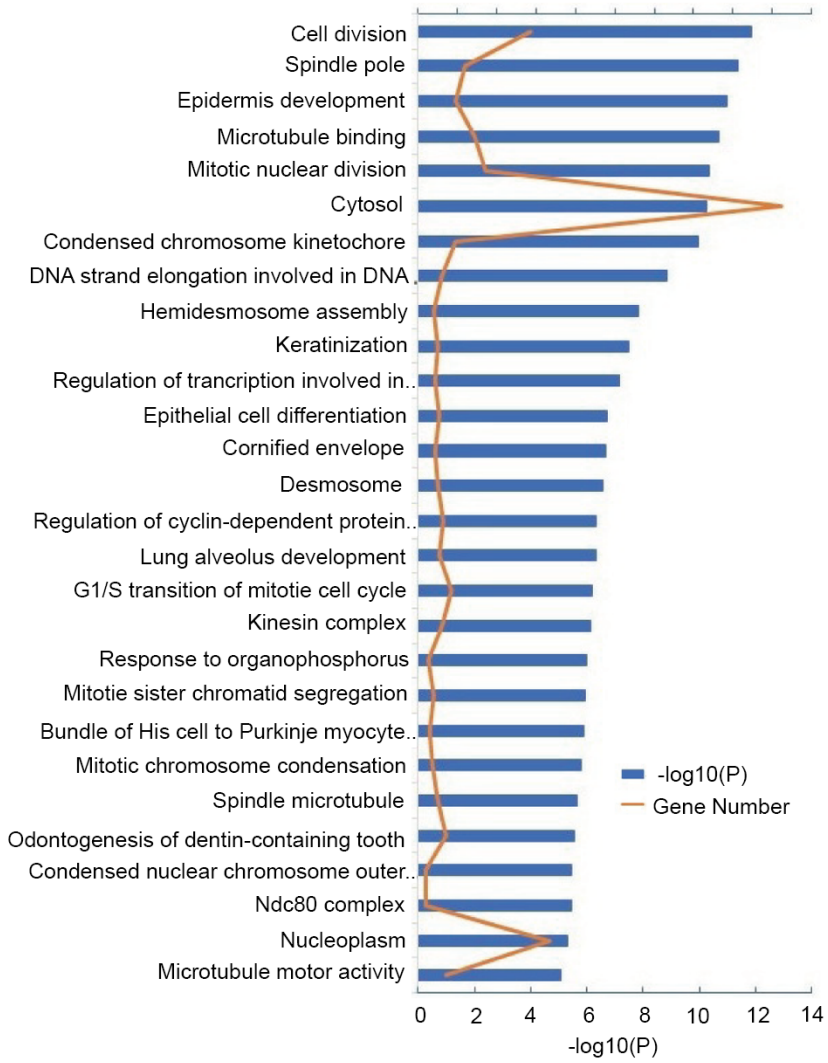

Figure 2. GO annotation analysis of differentially expressed mRNAs between LUAD and LUSC based on The Cancer Genome Atlas database. (A) Biological functions in LUAD and (B) LUSC. The blue column represents the degree of enrichment. The orange line represents the number of genes enriched. GO, Gene Ontology; LUAD, lung adenocarcinoma; LUSC, lung squamous cell carcinoma.

and SFTPB were selected for verification. A total of four differentially expressed exosomal mRNAs were selected for validation with serum samples from 70 patients with NSCLC (54 patients with LUAD and 16 patients with LUSC) using RT-qPCR. In order to ensure the accuracy of the results, ACTB and SLC25A6 were used as internal references in the present study. As shown in Fig. 5A and B, exosomal TP63 and KRT5 mRNA levels were significantly upregulated in patients with LUSC $(\mathrm{P}<0.01)$ compared with those in patients with LUAD. However, exosomal CEACAM6 and SFTPB mRNA levels were significantly upregulated in patients with LUAD compared with those in patients with LUSC, with ACTB as the internal control $(\mathrm{P}<0.01$ and $\mathrm{P}<0.05$, respectively; Fig. 5C and D). Similarly, as shown in Fig. 5E and F, exosomal TP63 and KRT5 mRNA levels were significantly upregulated in patients with LUSC $(\mathrm{P}<0.01)$ compared with those in patients with LUAD. However, exosomal CEACAM6 and SFTPB mRNA levels were significantly upregulated in patients with LUAD compared with those in patients with LUSC, with SLC25A6 as an internal control $(\mathrm{P}<0.01$ and $\mathrm{P}<0.05$, respectively; Fig. 5G and $\mathrm{H}$ ).

ROC curves were drawn to evaluate the clinical diagnostic value of four DEGs (TP63, KRT5, CEACAM6 and SFTPB) for patients with LUAD and LUSC. ROC curves were calculated using ACTB and SLC25A6 for modeling. The AUC of exosomal TP63 was 0.682 (95\% CI, 0.526-0.837), and the sensitivity and specificity were 74.1 and $62.5 \%$, respectively, based on ACTB (Fig. 6A). The AUC of exosomal KRT5 was 0.683 (95\% CI, 0.525-0.842), and the sensitivity and specificity were 79.6 and $62.5 \%$, respectively, based on ACTB (Fig. 6B). The AUC of exosomal CEACAM6 was 0.681 (95\% CI, 0.547-0.814), and the sensitivity and specificity were 87.5 and $53.7 \%$, respectively, based on ACTB (Fig. 6C). The AUC of exosomal SFTPB was 0.686 (95\% CI, 0.533-0.838), and the sensitivity and specificity were 43.7 and $90.7 \%$, respectively, based on ACTB (Fig. 6D). In addition, the combination of TP63, KRT5, CEACAM6 and SFTPB yielded an AUC of 0.757 (95\% CI, 0.625-0.889) with a sensitivity of $75.0 \%$ and specificity of $72.2 \%$, indicating a greater efficacy compared with that obtained by any one marker alone, based on ACTB (Fig. 6E). Similarly, the AUC of exosomal TP63 was 0.716 (95\% CI, 0.560-0.872), and the sensitivity and specificity were 61.1 and $75.0 \%$, respectively, based on SLC25A6 (Fig. 6F). The AUC of exosomal KRT5 was 0.715 (95\% CI, 0.586-0.844), and the sensitivity and specificity were 81.3 and $66.7 \%$, respectively, based on SLC25A6 (Fig. 6G). The AUC of exosomal CEACAM6 was 0.730 (95\% CI, 0.592-0.869), and the sensitivity and specificity were $81.3 \%$ and $66.7 \%$, respectively, based on SLC25A6 (Fig. 6H). The AUC of exosomal SFTPB was 0.701 (95\% CI, 0.548-0.855), and the sensitivity and specificity were 50.0 and $87.0 \%$, respectively, based on SLC25A6 (Fig. 6I). In addition, the combination of TP63, KRT5, CEACAM6 and SFTPB yielded an AUC of 0.822 (95\% CI, 0.699-0.945) with a sensitivity of $62.5 \%$ and specificity of $88.89 \%$, indicating 
A

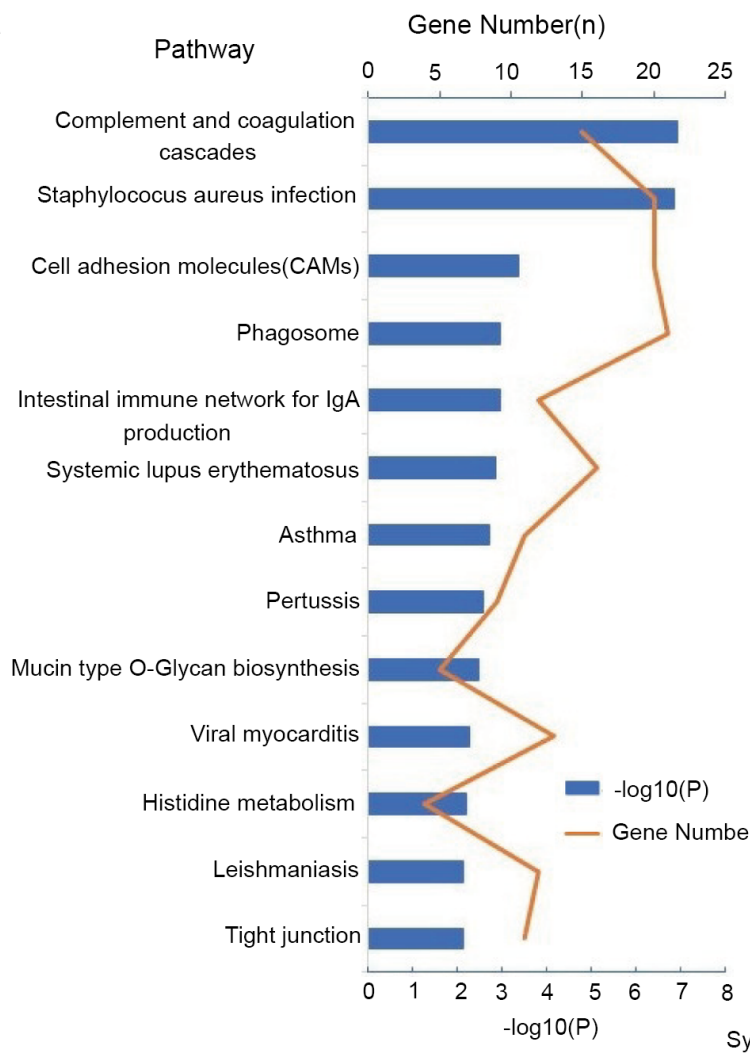

B

Pathway

Gene Number(n)

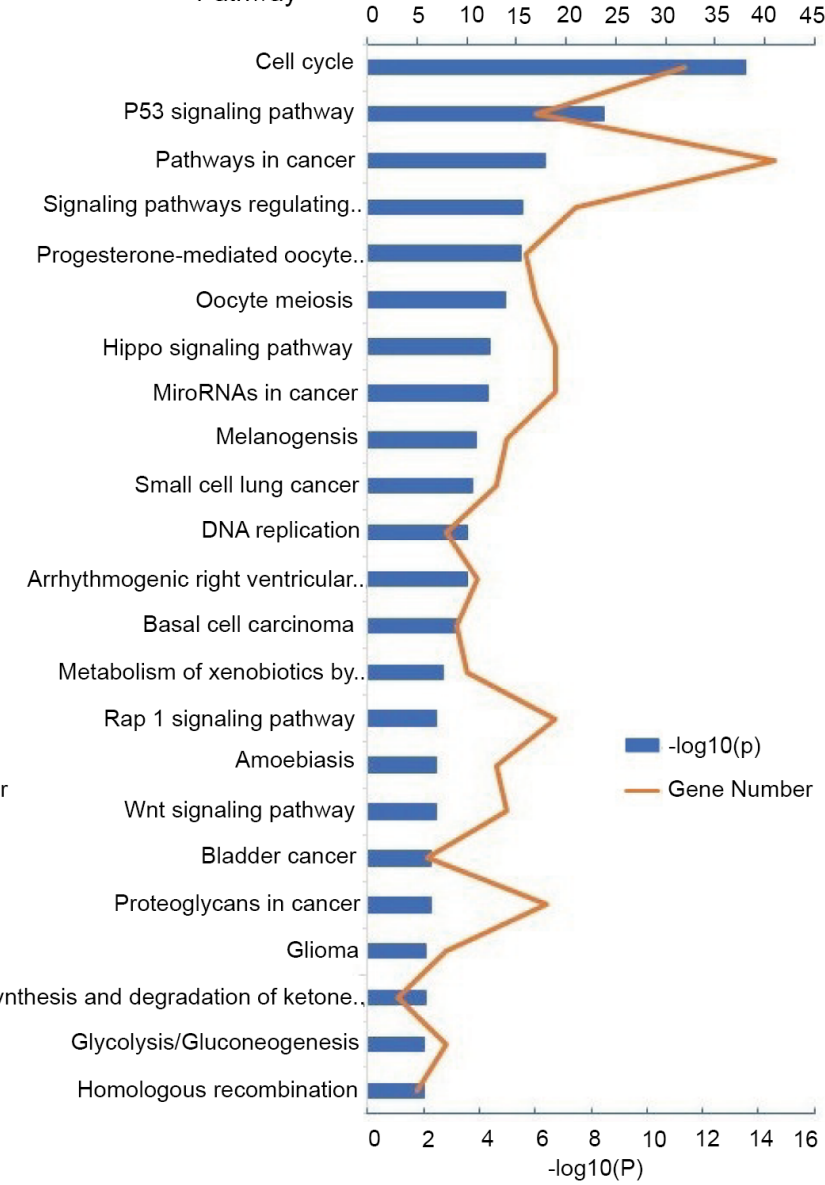

Figure 3. Kyoto Encyclopedia of Genes and Genomes pathway analysis of differentially expressed mRNAs between LUAD and LUSC based on The Cancer Genome Atlas database. (A) Tumor-related pathways in LUAD and (B) LUSC. The blue column represents the degree of enrichment. The orange line represents the number of genes enriched. LUAD, lung adenocarcinoma; LUSC, lung squamous cell carcinoma.

a greater efficacy compared with that obtained by any one marker alone, based on SLC25A6 (Fig. 6J).

Although two internal reference genes were used, the statistical results were basically consistent (Figs. 5 and 6), and the combined use of the four genes improved the ability to distinguish LUAD from LUSC. These aforementioned results demonstrated that exosomal TP63, KRT5, CEACAM6 and SFTPB mRNA were potential clinical diagnostic markers for distinguishing LUAD from LUSC.

\section{Discussion}

Recently, a number of studies have demonstrated that exosomes can carry biomarkers, and have diagnostic and prognostic value (45-47). Although certain studies have demonstrated that mRNAs in peripheral blood can be used as biomarkers for the clinical diagnosis of LUAD and LUSC $(48,49)$, to the best of our knowledge, there is currently no relevant research available on the mRNAs from exosomes. In the present study, exosomes were isolated from patient serum and the diagnostic value of exosomes as biomarkers to distinguish LUAD from LUSC was investigated. Due to the endocytic origin of exosomes, the composition of exosomes reflects the composition of parental cells; exosomes represent potential substitutes for primitive cells (50). It is well known that exosomes are stable and are relatively difficult to degrade, and can be used to identify original cells (51). The formation of exosomes is associated with cell secretion and involves a variety of proteins, such as Rab proteins (Rab27A/B), heat shock protein 70, TSG101, Alix and tetraspanins (CD9, CD63, CD81 and CD82); these proteins are considered to be the exosome markers for identifying true exosomes $(50,52)$. As exosomes can be easily obtained and identified from most body fluids, they may become a promising biomarker for lung cancer (53). In the present study, Alix, TSG101 and CD63 were selected as markers for identifying true exosomes. The results revealed that the exosome markers were positive, indicating that exosomes were successfully extracted.

It has been shown that multiple genes can be used as potential biomarkers for the diagnosis and prognosis of lung cancer $(54,55)$. However, to the best of our knowledge, there are currently limited studies available on the effective molecular diagnostic markers of LUAD and LUSC. Based on TCGA database, the present study identified eight DEGs that were overexpressed in patients with LUSC compared with those with LUAD; and nine DEGs that were downregulated in patients with LUSC compared with those with LUAD.

In addition, KEGG and GO analyses were performed on these DEGs. The results demonstrated that LUAD was associated with the GO term 'BMP receptor binding'. Bone 
A

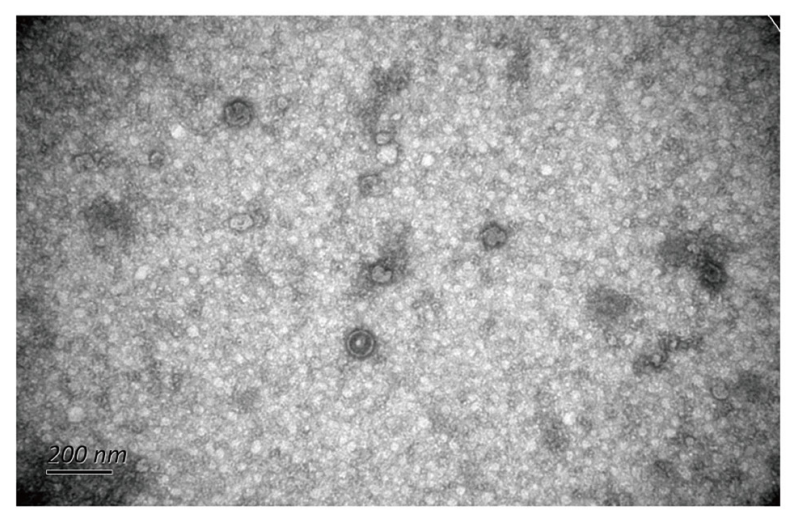

C

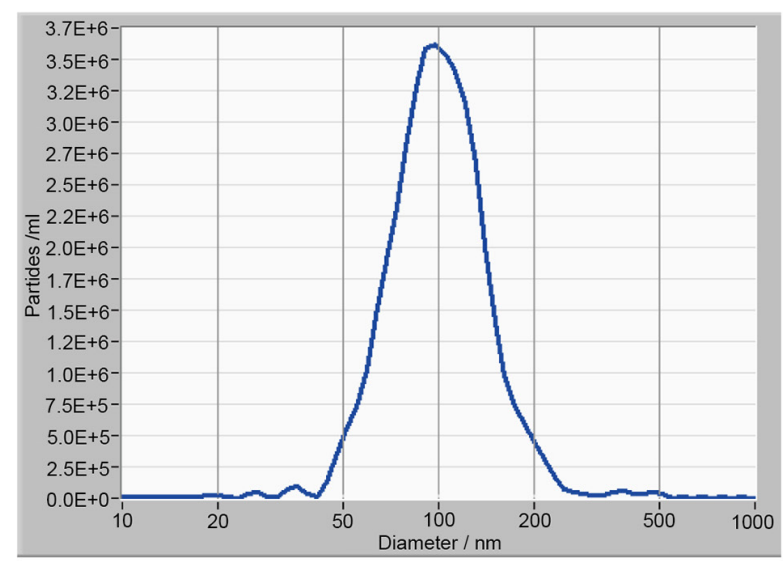

B
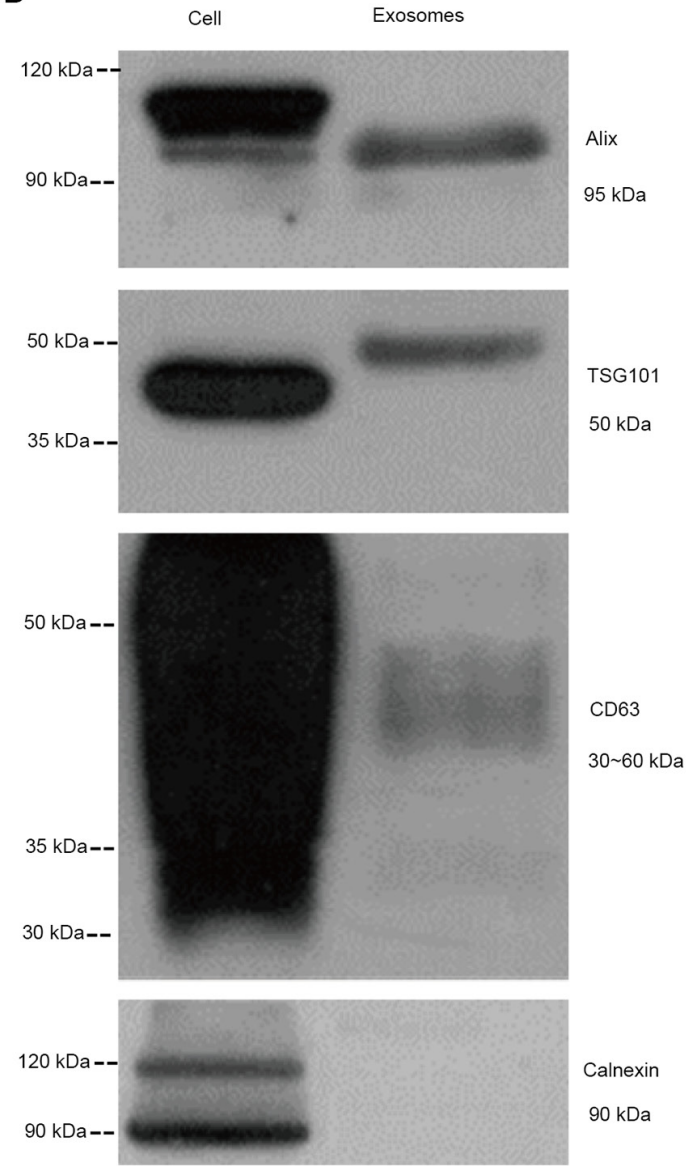

Figure 4. Characterization and quantification of exosomes. (A) The representative samples of transmission electron microscopic view of exosomes in patients with lung cancer. (B) The representative exosomes isolated from the peripheral blood of lung cancer tissue were examined by western blot analysis for the exosomal markers, Alix, TSG101 and CD63. Calnexin was used as a negative control. Alix, ALG-2 interacting protein X; TSG101, tumor susceptibility gene 101 protein. (C) The representative samples of size distribution of isolated exosomes using a nanoparticle tracking analyzer.

morphogenetic proteins (BMPs) belong to the transforming growth factor $\beta$ superfamily (56). Deng et al (57) observed that the mRNA level of BMP5 was higher in LUAD tissue compared with that in LUSC tissue. In the present study, 'Tight junction' was obviously enriched in LUAD, in both GO and KEGG analyses. Tight junctions belong to epithelial cells when forming intercellular junction complexes (58). Claudins are major components of tight junctions (59), and Claudin-2 is highly expressed in LUAD tissues and increases cell proliferation (60). In the present study, LUSC was associated with the GO terms 'Spindle pole' and 'Spindle microtubule'. Abnormal spindle-like microcephaly-associated protein (ASPM) is a type of microtubule-associated centrosome protein, which plays an important role in cell development (61). ASPM is mainly expressed in the ventricular zone of posterior fossa, and participates in the functional regulation of spindle tissue and cytokinesis (62). Yuan et al (63) demonstrated that ASPM promoted the progression of LUSC by targeting CDK4. The present study demonstrated that the p53 signaling pathway was upregulated in LUSC. The key role of p53 mutation in malignant transformation, histological progress, invasion and metastasis of lung cancer has been confirmed in lung cancer models in vitro and in vivo $(64,65)$. Smoking is closely associated with p53 mutation (66), which may explain the universality of p53 alterations in LUSC. These results suggest that DEGs affect the development of LUSC and LUAD via different pathways. Therefore, the screened DEGs can be used as markers to distinguish LUSC from LUAD.

Considering the expression level and relatively high specificity of exosome-derived DEGs in peripheral blood, TP63, KRT5, CEACAM6 and SFTPB were selected for follow-up experiments. TP63 and KRT5 levels were more concentrated in LUSC cases. It is well known that TP63 is a biomarker of squamous cell carcinoma $(67,68)$. The results of the present study revealed that TP63 expression in LUSC was significantly higher compared with that in LUAD. It has been demonstrated that TP63 can regulate cell proliferation and plays a key role in squamous cell carcinoma $(69,70)$. The results indicated that cell cycle-related molecular pathways play an important role in the development of squamous cell carcinoma. KRT5 protein is mainly expressed in basal keratinocytes of the epidermis and its overexpression is a unique feature of squamous cell carcinoma (32). Similar results were also obtained in a recent study (71). The aforementioned results provide a theoretical basis for the use of TP63 and KRT5 as clinical biomarkers for the diagnosis of LUSC. The expression of CEACAM6 and SFTPB from exosomes was higher in LUAD compared with in LUSC. It has been demonstrated that CEACAM6 is expressed at low levels in laryngeal squamous cell carcinoma cell lines (72), 

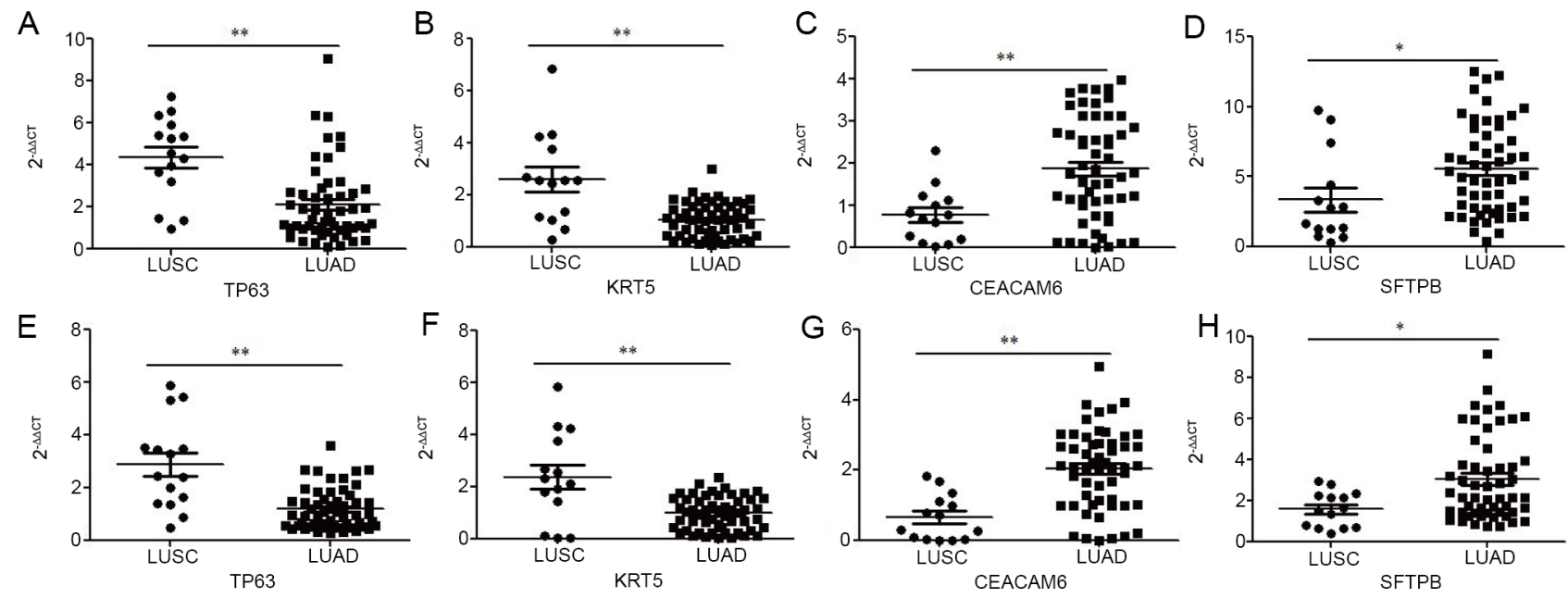

Figure 5. Relative expression of TP63, KRT5, CEACAM6 and SFTPB from exosomes isolated from two types of non-small cell lung cancer measured by RT-qPCR. (A) TP63 based on ACTB; (B) KRT5 based on ACTB; (C) CEACAM6 based on ACTB; (D) SFTPB based on ACTB; (E) TP63 based on SLC25A6; (F) KRT5 based on SLC25A6; (G) CEACAM6 based on SLC25A6; (H) SFTPB based on SLC25A6. "P<0.05; *"P<0.01; TP63, tumor protein P63; KRT5, keratin 5; CEACAM6, CEA cell adhesion molecule 6; SFTPB, surfactant protein B; ACTB, $\beta$-actin; SLC25A6, solute carrier family 25 member 6; RT-qPCR, reverse transcription-quantitative polymerase chain reaction; LUSC, lung squamous cell carcinoma; LUAD, lung adenocarcinoma.
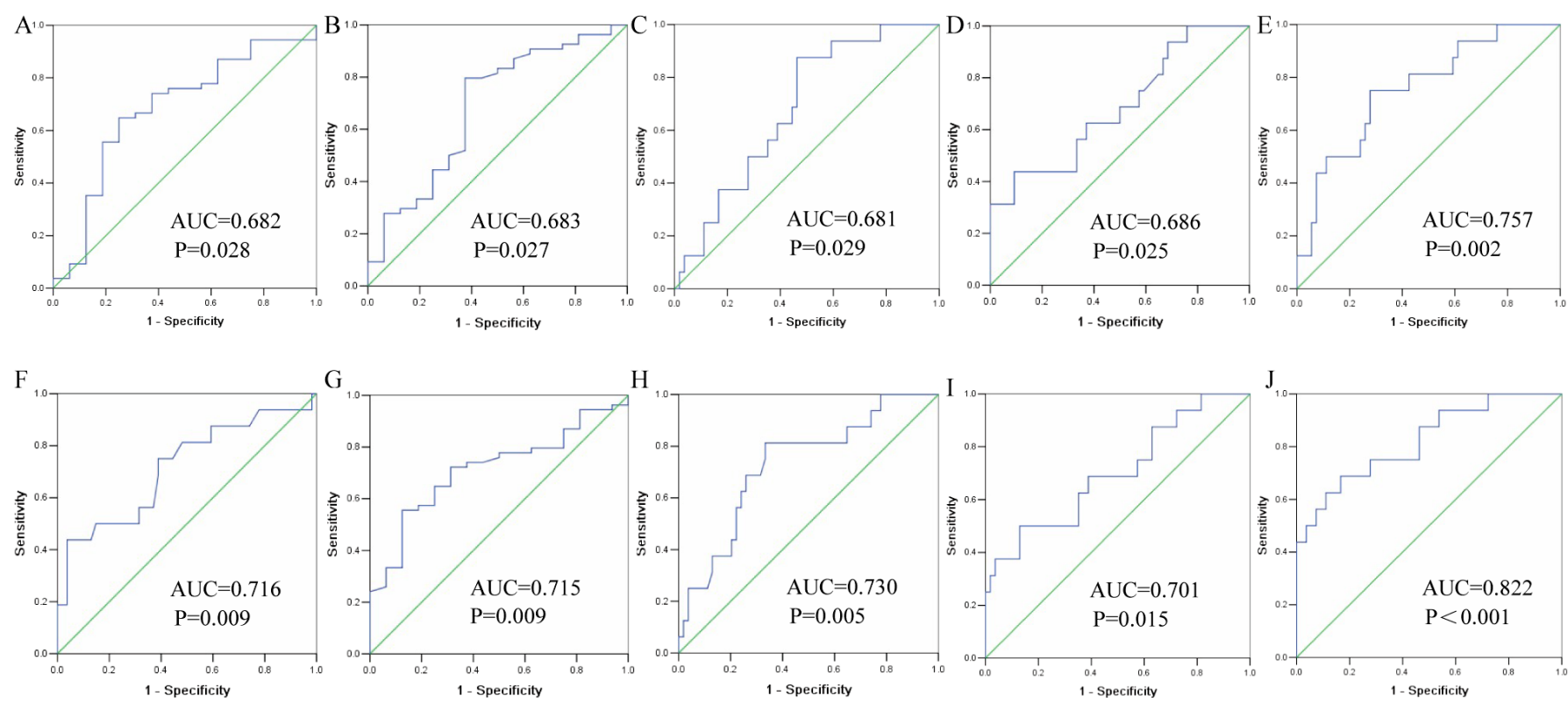

Figure 6. Receiving operator characteristic curve of four exosomal DEGs in distinguishing between LADC and LSCC. (A) TP63 based on ACTB; (B) KRT5 based on ACTB; (C) CEACAM6 based on ACTB; (D) SFTPB based on ACTB; (E) four DEGs based on ACTB; (F) TP63 based on SLC25A6; (G) KRT5 based on SLC25A6; (H) CEACAM6 based on SLC25A6; (I) SFTPB based on SLC25A6; (J) four DEGs based on SLC25A6. LUAD, lung adenocarcinoma; LUSC, lung squamous cell carcinoma; AUC, area under the curve; DEGs, differentially expressed genes; TP63, tumor protein P63; KRT5, keratin 5; CEACAM6, CEA cell adhesion molecule 6; SFTPB, surfactant protein B; ACTB, $\beta$-actin; SLC25A6, solute carrier family 25 member 6.

whereas it is expressed at high levels in prostate cancer and gastric adenocarcinoma $(73,74)$. Hong et al (39) found CEACAM6 protein expression in $85.7 \%$ of 70 LUAD tissues, whereas all LUSC tissues stained negative for CEACAM6, which is consistent with the findings of previous studies. Borczuk et al (75) demonstrated that SFTPB, a member of lung-specific transcription pathways, played a role in the development of LUAD. These findings correspond with the results of the present study. At least in part, it was confirmed that the four DEGs selected herein may be used as markers to distinguish LUSC from LUAD.
In clinical practice, certain patients are highly suspected as having malignant tumors or have been confirmed to have malignant tumors; however, this cannot be further classified. In clinical practice, it is difficult to obtain tumor tissues, or only a small number of pathological specimens are obtained, which is insufficient for pathological diagnosis as the whole pathological tissue includes necrotic tissue that cannot be stained by immunohistochemistry. In addition, the lipid bilayer of exosomes allows for stable cargo, which are relatively difficult to degrade (76). If biomarkers for distinguishing pathological types in peripheral blood were detected accurately, this method 
would improve the efficiency and accuracy of diagnosis, and may provide clinical guidance for these patients.

In conclusion, it is evident that TP63, KRT5, CEACAM6 and SFTPB derived from exosomes may be used as potential blood biomarkers for distinguishing LUAD from LUSC. The combination of multiple biomarkers may improve the specificity and sensitivity of the diagnosis of different lung cancer subtypes. Due to the imbalance in LUAD and LUSC sample numbers, the statistical results may be deviated. The authors aim to further expand the sample size and perform a multicenter study in order to investigate the potential use of these biomarkers in the diagnosis of LUAD and LUSC.

\section{Acknowledgements}

Not applicable.

\section{Funding}

No funding was received.

\section{Availability of data and materials}

The datasets used and/or analyzed during the present study are available from the corresponding author upon reasonable request.

\section{Authors' contributions}

$\mathrm{BC}$ and JL contributed to the experimental design. $\mathrm{BC}$ and PW performed some of the experiments. BC and LG made contributions to acquisition and analysis of data. $\mathrm{BC}$ analyzed the data and drafted the initial manuscript. BC and JL confirm the authenticity of all the raw data. All authors have read and approved the final manuscript.

\section{Ethics approval and consent to participate}

The present study was approved by the Ethics Committee of the Fourth Hospital of Hebei Medical University. Informed consent was signed by all patients or their families. If the patient died or had other incapacity, a family member signed the consent form.

\section{Patient consent for publication}

Not applicable.

\section{Competing interests}

The authors declare that they have no competing interests.

\section{References}

1. Bray F, Ferlay J, Soerjomataram I, Siegel RL, Torre LA and Jemal A: Global cancer statistics 2018: GLOBOCAN estimates of incidence and mortality worldwide for 36 cancers in 185 countries. CA Cancer J Clin 68: 394-424, 2018.

2. Otsmane A, Kacimi G, Adane S, Cherbal F and Aouichat Bouguerra S: Clinico-epidemiological profile and redox imbalance of lung cancer patients in Algeria. J Med Life 11: 210-217, 2018.
3. Yang H, Lin Y and Liang Y: Treatment of lung carcinosarcoma and other rare histologic subtypes of non-small cell lung cancer. Curr Treat Options Oncol 18: 54, 2017.

4. Lim SL, Jia Z, Lu Y, Zhang H, Ng CT, Bay BH, Shen HM and Ong CN: Metabolic signatures of four major histological types of lung cancer cells. Metabolomics 14: 118, 2018.

5. Björkqvist AM, Husgafvel-Pursiainen K, Anttila S, Karjalainen A, Tammilehto L, Mattson K, Vainio H and Knuutila S: DNA gains in $3 q$ occur frequently in squamous cell carcinoma of the lung, but not in adenocarcinoma. Genes Chromosomes Cancer 22: 79-82, 1998.

6. Cooper WA, Lam DC, O'toole SA and Minna JD: Molecular biology of lung cancer. J Thorac Dis 5 (Suppl 5): S479-S490, 2013.

7. Davidson MR, Gazdar AF and Clarke BE: The pivotal role of pathology in the management of lung cancer. J Thorac Dis 5 (Suppl 5): S463-S478, 2013.

8. Langer CJ, Besse B, Gualberto A, Brambilla E and Soria JC: The evolving role of histology in the management of advanced non-small-cell lung cancer. J Clin Oncol 28: 5311-5320, 2010

9. Tomasini P, Khobta N, Greillier L and Barlesi F: Ipilimumab: Its potential in non-small cell lung cancer. Ther Adv Med Oncol 4: 43-50, 2012.

10. Paez JG, Jänne PA, Lee JC, Tracy S, Greulich H, Gabriel S, Herman P, Kaye FJ, Lindeman N, Boggon TJ, et al: EGFR mutations in lung cancer: Correlation with clinical response to gefitinib therapy. Science 304: 1497-1500, 2004.

11. Xiao J, Lu X, Chen X, Zou Y, Liu A, Li W, He B, He S and Chen Q: Eight potential biomarkers for distinguishing between lung adenocarcinoma and squamous cell carcinoma. Oncotarget 8: 71759-71771, 2017.

12. Liu F, Vermesh O, Mani V, Ge TJ, Madsen SJ, Sabour A, Hsu EC, Gowrishankar G, Kanada M, Jokerst JV, et al: The exosome total isolation chip. ACS Nano 11: 10712-10723, 2017.

13. Fitts CA, Ji N, Li Y and Tan C: Exploiting exosomes in cancer liquid biopsies and drug delivery. Adv Healthc Mater 8: e1801268, 2019.

14. Carretero-González A, Otero I, Carril-Ajuria L, de Velasco G and Manso L: Exosomes: Definition, role in tumor development and clinical implications. Cancer Microenviron 11: 13-21, 2018.

15. Chuo ST, Chien JC and Lai CP: Imaging extracellular vesicles: Current and emerging methods. J Biomed Sci 25: 91, 2018.

16. Revenfeld ALS Bæk R, Nielsen MH, Stensballe A, Varming K and Jørgensen M: Diagnostic and prognostic potential of extracellular vesicles in peripheral blood. Clin Ther 36: 830-846, 2014.

17. Robinson MD, McCarthy DJ and Smyth GK: edgeR: A bioconductor package for differential expression analysis of digital gene expression data. Bioinformatics 26: 139-140, 2010.

18. Wang N, Song X, Liu L, Niu L, Wang X, Song X and Xie L: Circulating exosomes contain protein biomarkers of metastatic non-small-cell lung cancer. Cancer Sci 109: 1701-1709, 2018.

19. Livak KJ and Schmittgen TD: Analysis of relative gene expression data using real-time quantitative PCR and the 2(-Delta Delta C(T)) method. Methods 25: 402-408, 2001.

20. Bustin SA: Quantification of mRNA using real-time reverse transcription PCR (RT-PCR): Trends and problems. J Mol Endocrinol 29: 23-39, 2002.

21. Vandesompele J, De Preter K, Pattyn F, Poppe B, Van Roy N, De Paepe A and Speleman F: Accurate normalization of real-time quantitative RT-PCR data by geometric averaging of multiple internal control genes. Genome Biol 3: RESEARCH0034, 2002.

22. Lourenço AP, Mackert A, Cristino ADS and Simões ZLP: Validation of reference genes for gene expression studies in the honey bee, Apis mellifera, by quantitative real-time RT-PCR. Apidologie 39: 372-385, 2008.

23. Macabelli CH, Ferreira RM, Gimenes LU, de Carvalho NA, Soares JG, Ayres H, Ferraz ML, Watanabe YF, Watanabe OY, Sangalli JR, et al: Reference gene selection for gene expression analysis of oocytes collected from dairy cattle and buffaloes during winter and summer. PLoS One 9: e93287, 2014.

24. Dong ZZ, Tang CH, Zhang Z, Zhou W, Zhao R, Wang L, Xu JC, Wu YY, Wu J,Zhang X, et al: Simultaneous detection of exosomal membrane protein and RNA by highly sensitive aptamer assisted multiplex-PCR. ACS Appl Bio Mater 3: 2560-2567, 2020.

25. Pan H, Yang XW, Bidne K, Hellmich RL, Siegfried BD and Zhou XG: Selection of reference genes for RT-qPCR analysis in the monarch butterfly, Danaus plexippus (L.), a migrating bio-indicator. PLoS One 10: e0129482, 2015. 
26. Zhang S, An S, Li Z, Wu F, Yang Q, Liu Y, Cao J, Zhang H, Zhang $Q$ and Liu X: Identification and validation of reference genes for normalization of gene expression analysis using qRT-PCR in Helicoverpa armigera (Lepidoptera: Noctuidae). Gene 555: 393-402, 2015.

27. Benjamini Y and Hochberg Y: Controlling the false discovery rate: A practical and powerful approach to multiple testing. J Royal Stat Soc B 57: 289-300, 1995.

28. Liu P, Wang H, Liang Y, Hu A, Xing R, Jiang L, Yi L and Dong J: LINC00852 promotes lung adenocarcinoma spinal metastasis by targeting S100A9. J Cancer 9: 4139-4149, 2018

29. Stewart PA, Welsh EA, Slebos RJC, Fang B, Izumi V, Chambers M Zhang G, Cen L, Pettersson F, Zhang Y, et al: Proteogenomic landscape of squamous cell lung cancer. Nat Commun 10: 3578, 2019.

30. Kim Y, Shiba-Ishii A, Nakagawa T, Iemura SI, Natsume T, Nakano N, Matsuoka R, Sakashita S, Lee S, Kawaguchi A, et al: Stratifin regulates stabilization of receptor tyrosine kinases via interaction with ubiquitin-specific protease 8 in lung adenocarcinoma. Oncogene 37: 5387-5402, 2018

31. Wang Z, Yang MQ, Lei L, Fei LR, Zheng YW, Huang WJ, Li ZH, Liu CC and Xu HT: Overexpression of KRT17 promotes proliferation and invasion of non-small cell lung cancer and indicates poor prognosis. Cancer Manag Res 11: 7485-7497, 2019.

32. Khayyata S, Yun S, Pasha T, Jian B, McGrath C, Yu G, Gupta P and Baloch Z: Value of P63 and CK5/6 in distinguishing squamous cell carcinoma from adenocarcinoma in lung fine-needle aspiration specimens. Diagn Cytopathol 37: 178-183, 2009.

33. Yang B, Zhang W, Zhang M, Wang X, Peng S and Zhang R: KRT6A promotes EMT and cancer stem cell transformation in lung adenocarcinoma. Technol Cancer Res Treat 19: $1533033820921248,2020$.

34. Konda JD, Olivero M, Musiani D, Lamba S and Di Renzo MF: Heat-shock protein 27 (HSP27, HSPB1) is synthetic lethal to cells with oncogenic activation of MET, EGFR and BRAF. Mo Oncol 11: 599-611, 2017.

35. Ohtsuka T, Sakaguchi M, Yamamoto H, Tomida S, Takata K, Shien K, Hashida S, Miyata-Takata T, Watanabe M Suzawa K, et al: Interaction of cytokeratin 19 head domain and HER 2 in the cytoplasm leads to activation of HER2-Erk pathway. Sci Rep 6: 39557, 2016.

36. Song Y, Sun Y, Lei Y, Yang K and Tang R: YAP1 promotes multidrug resistance of small cell lung cancer by CD74-related signaling pathways. Cancer Med 9: 259-268, 2020.

37. Tokumoto H: Analysis of HLA-DRB1-related alleles in Japanese patients with lung cancer-relationship to genetic susceptibility and resistance to lung cancer. J Cancer Res Clin Oncol 124: 511-516, 1998.

38. Martins I, Deshayes F, Baton F, Forget A, Ciechomska I, Sylla K, Aoudjit F, Charron D, Al-Daccak R and Alcaide-Loridan C: Pathologic expression of MHC class II is driven by mitogenactivated protein kinases. Eur J Immunol 37: 788-797, 2007.

39. Hong KP, Shin MH, Yoon S, Ji GY, Moon YR, Lee OJ, Choi SY, Lee YM, Koo JH, Lee HC, et al: Therapeutic effect of ant CEACAM6 monoclonal antibody against lung adenocarcinoma by enhancing anoikis sensitivity. Biomaterials 67: 32-41, 2015.

40. Wang Y, Yang W, Pu Q, Yang Y, Ye S, Ma Q, Ren J, Cao Z, Zhong G, Zhang $\mathrm{X}$, et al: The effects and mechanisms of SLC34A2 in tumorigenesis and progression of human non-small cell lung cancer. J Biomed Sci 22: 52, 2015.

41. Lee S, Kim D, Kang J, Kim E, Kim W, Youn H and Youn B: Surfactant protein B suppresses lung cancer progression by inhibiting secretory phospholipase A2 activity and arachidonic acid production. Cell Physiol Biochem 42: 1684-1700, 2017.

42. Hasegawa Y, Takahashi M, Ariki S, Saito A, Uehara Y Takamiya R, Kuronuma K, Chiba H, Sakuma Y, Takahashi H and Kuroki Y: Surfactant protein A down-regulates epidermal growth factor receptor by mechanisms different from those of surfactant protein D. J Biol Chem 292: 18565-18576, 2017.

43. Wang Y, Kuan PJ, Xing C, Cronkhite JT, Torres F, Rosenblatt RL, DiMaio JM, Kinch LN, Grishin NV and Garcia CK: Genetic defects in surfactant protein A2 are associated with pulmonary fibrosis and lung cancer. Am J Hum Genet 84: 52-59, 2009.

44. Min L, Zhu S, Chen L, Liu X, Wei R, Zhao L, Yang Y, Zhang Z, Kong G, Li P and Zhang S: Evaluation of circulating small extracellular vesicles derived miRNAs as biomarkers of early colon cancer: A comparison with plasma total miRNAs. J Extracell Vesicles 8: 1643670, 2019.

45. Li Y, Yin Z, Fan J, Zhang S and Yang W: The roles of exosomal miRNAs and lncRNAs in lung diseases. Signal Transduct Target Ther 4: 47, 2019.
46. Li C, Li C, Zhi C, Liang W, Wang X, Chen X, Lv T, Shen Q, Song Y, Lin D and Liu H: Clinical significance of PD-L1 expression in serum-derived exosomes in NSCLC patients. J Transl Med 17: 355, 2019.

47. Chen R, Xu X, Qian Z, Zhang C, Niu Y, Wang Z, Sun J, Zhang X and $\mathrm{Yu} \mathrm{Y}$ : The biological functions and clinical applications of exosomes in lung cancer. Cell Mol Life Sci 76: 4613-4633, 2019.

48. Fu L, Wang H, Wei D, Wang B, Zhang C, Zhu T, Ma Z, Li Z, Wu Y and $\mathrm{Yu}$ G: The value of CEP55 gene as a diagnostic biomarker and independent prognostic factor in LUAD and LUSC. PLoS One 15: $\mathrm{e} 0233283,2020$

49. Wang C, Tan S, Liu WR, Lei Q, Qiao W, Wu Y, Liu X, Cheng W, Wei YQ, Peng Y and Li W: RNA-Seq profiling of circular RNA in human lung adenocarcinoma and squamous cell carcinoma. Mol Cancer 18: 134, 2019

50. Kowal J, Tkach M and Théry C: Biogenesis and secretion of exosomes. Curr Opin Cell Biol 29: 116-125, 2014.

51. Molina-Vila MA, Mayo-de-Las-Casas C, Giménez-Capitán A, Jordana-Ariza N, Garzón M, Balada A, Villatoro S, Teixidó C, García-Peláez B, Aguado C, et al: Liquid biopsy in non-small cell lung cancer. Front Med (Lausanne) 3: 69, 2016.

52. Yoshioka Y, Konishi Y, Kosaka N, Katsuda T, Kato T and Ochiya T: Comparative marker analysis of extracellular vesicles in different human cancer types. J Extracell Vesicles: Jun 18, 2013 (Epub ahead of print). do: 10.3402/jev.v2i0.20424 2013.

53. Vlassov AV, Magdaleno S, Setterquist R and Conrad R: Exosomes: Current knowledge of their composition, biological functions, and diagnostic and therapeutic potentials. Biochim Biophys Acta 1820: 940-948, 2012

54. Wang K, Chen R, Feng Z, Zhu YM, Sun XX, Huang W and Chen $\mathrm{ZN}$ : Identification of differentially expressed genes in non-small cell lung cancer. Aging (Albany NY) 11: 11170-11185, 2019.

55. Mehta A, Sriramanakoppa NN, Agarwal P, Viswakarma G, Vasudevan S, Panigrahi M, Kumar D, Saifi M, Chowdhary I, Doval DC and Suryavanshi M: Predictive biomarkers in nonsmall cell carcinoma and their clinico-pathological association. South Asian J Cancer 8: 250-254, 2019.

56. Miyazono K, Kamiya Y and Morikawa M: Bone morphogenetic protein receptors and signal transduction. J Biochem 147: 35-51, 2010.

57. Deng T, Lin D, Zhang M, Zhao Q, Li W, Zhong B, Deng Y and $\mathrm{Fu} X$ : Differential expression of bone morphogenetic protein 5 in human lung squamous cell carcinoma and adenocarcinoma. Acta Biochim Biophys Sin (Shanghai) 47: 557-563, 2015.

58. Powell DW: Barrier function of epithelia. Am J Physiol 241: G275-G288, 1981.

59. Tsukita S, Furuse $M$ and Itoh M: Multifunctional strands in tight junctions. Nat Rev Mol Cell Biol 2: 285-293, 2001.

60. Hichino A, Okamoto M, Taga S, Akizuki R, Endo S, Matsunaga T and Ikari A: Down-regulation of Claudin-2 expression and proliferation by epigenetic inhibitors in human lung adenocarcinoma A549 cells. J Biol Chem 292: 2411-2421, 2017.

61. Pai VC, Hsu CC, Chan TS, Liao WY, Chuu CP, Chen WY, Li CR, Lin CY, Huang SP, Chen LT and Tsai KK: ASPM promotes prostate cancer stemness and progression by augmenting Wnt-Dvl-3- $\beta$-catenin signaling. Oncogene 38: 1340-1353, 2019.

62. Gai M, Bianchi FT, Vagnoni C, Vernì F, Bonaccorsi S, Pasquero S, Berto GE, Sgrò F, Chiotto AA, Annaratone L, et al: ASPM and CITK regulate spindle orientation by affecting the dynamics of astral microtubules. EMBO Rep 18: 1870, 2017.

63. Yuan YJ, Sun Y, Gao R, Yin ZZ, Yuan ZY and Xu LM: Abnormal spindle-like microcephaly-associated protein (ASPM) contributes to the progression of lung squamous cell carcinoma (LSCC) by regulating CDK4. J Cancer 11: 5413-5423, 2020.

64. Kemp CJ, Donehower LA, Bradley A and Balmain A: Reduction of p53 gene dosage does not increase initiation or promotion but enhances malignant progression of chemically induced skin tumors. Cell 74: 813-822, 1993.

65. Jackson EL, Olive KP, Tuveson DA, Bronson R, Crowley D, Brown $\mathrm{M}$ and Jacks T: The differential effects of mutant p53 alleles on advanced murine lung cancer. Cancer Res 65: 10280-10288, 2005.

66. Gibbons DL, Byers LA and Kurie JM: Smoking, p53 mutation, and lung cancer. Mol Cancer Res 12: 3-13, 2014.

67. Yang Z, Zhuan B, Yan Y, Jiang S and Wang T: Integrated analyses of copy number variations and gene differential expression in lung squamous-cell carcinoma. Biol Res 48: 47, 2015.

68. Zhang S, Li M, Ji H and Fang Z: Landscape of transcriptional deregulation in lung cancer. BMC Genomics 19: 435, 2018. 
69. Cheng H, Yang X, Si H, Saleh AD, Xiao W, Coupar J, Gollin SM, Ferris RL, Issaeva N, Yarbrough WG, et al: Genomic and transcriptomic characterization links cell lines with aggressive head and neck cancers. Cell Rep 25: 1332-1343.e5, 2018.

70. Yoshida M, Yokota E, Sakuma T, Yamatsuji T, Takigawa N, Ushijima T, Yamamoto T, Fukazawa $\mathrm{T}$ and Naomoto Y: Development of an integrated CRISPRi targeting $\triangle \mathrm{Np} 63$ for treatment of squamous cell carcinoma. Oncotarget 9: 29220-29232, 2018.

71. Li X, Shi G, Chu Q, Jiang W, Liu Y, Zhang S, Zhang Z, Wei Z, $\mathrm{He}$ F, Guo Z and Qi L: A qualitative transcriptional signature for the histological reclassification of lung squamous cell carcinomas and adenocarcinomas. BMC Genomics 20: 881, 2019.

72. Bednarek K, Kostrzewska-Poczekaj M, Szaumkessel M, Kiwerska K, Paczkowska J, Byzia E, Ustaszewski A, Janiszewska J, Bartochowska A, Grenman R, et al: Downregulation of CEACAM6 gene expression in laryngeal squamous cell carcinoma is an effect of DNA hypermethylation and correlates with disease progression. Am J Cancer Res 8: 1249-1261, 2018.
73. Duxbury MS, Matros E, Clancy T, Bailey G, Doff M, Zinner MJ, Ashley SW, Maitra A, Redston M and Whang EE: CEACAM6 is a novel biomarker in pancreatic adenocarcinoma and PanIN lesions. Ann Surg 241: 491-496, 2005.

74. Zang M, Zhang Y, Zhang B, Hu L, Li J, Fan Z, Wang H, Su L, Zhu Z, Li C, et al: CEACAM6 promotes tumor angiogenesis and vasculogenic mimicry in gastric cancer via FAK signaling. Biochim Biophys Acta 1852: 1020-1028, 2015.

75. Borczuk AC, Gorenstein L, Walter KL, Assaad AA, Wang L and Powell CA: Non-small-cell lung cancer molecular signatures recapitulate lung developmental pathways. Am J Pathol 163: 1949-1960, 2003.

76. Cui S, Cheng Z, Qin W and Jiang L: Exosomes as a liquid biopsy for lung cancer. Lung Cancer 116: 46-54, 2018. International (CC BY-NC-ND 4.0) License. 